\author{
L.N. Gumilyov Eurasian National University, Astana, Kazakhstan \\ (E-mail: jetpisov_k54@mail.ru)
}

\title{
Analytical and graphical methods for the solution of one problem of transport logistics
}

\begin{abstract}
This article shows the way to solve a specific problem of transport logistics using the potential method. The first support plan is constructed by the method of the north-western corner. The article also shows a graphical method for solving the problem. The article shows the optimal method for solving the problem of transport logistics using the example of the real economy of the Kostanay region (Arkalyk).
\end{abstract}

Keywords: Logistics, transport task, potencial, northwest corner, count, bipartite count, value, volume.

Logistics transportation - a system for organizing the movement of goods, with the choice of the optimal route, reducing the time and costs money.

Cargo transportation takes over the entire range of organizational issues in the field of transport logistics related to the transportation and safety products [1].

Logistics - the science of the movement of goods, controlled by a material flow and information [2].

Transport logistics - all steps from planning to organization and control of cargo transportation from getting to the final place of delivery of the item and from the source to consumer [3].

transport logistics tasks consist of:

- choosing the type and mode of transport;

- planning of transport processes;

- ensuring the unity of the whole process;

- preparation of optimal transportation routes.

To determine the total cost of transportation (S), it is necessary to know:

1) Fuel consumption for 1 liter;

2) The salary of the driver;

3) Amortization.

In this paper, we solve the problem of logistics below these farms, Kostanay region, Arkalyk city. Name of farms, the volume of the harvest and transportation, and storage in the following table (Table 1, 2). It also indicated the distance between farms and granaries (Table 3). Fuel consumption depends on the distance and type (load) technology.

Volumes of goods at the points of departure

\begin{tabular}{|c|c|c|c|c|}
\hline Title grain farms & $\begin{array}{c}\text { Square crop } \\
\text { (sown) }(\text { ha) }\end{array}$ & $\begin{array}{c}\text { Collected } \\
\text { wheat }(\mathrm{T})\end{array}$ & $\begin{array}{c}\text { Productivity } \\
\text { per hectare }(\mathrm{c})\end{array}$ & $\begin{array}{c}\text { Number } \\
\text { of flights }\end{array}$ \\
\hline Aiman $A_{1}$ & 800 & 400 & 5 & 17 \\
\hline Sharipa $A_{2}$ & 500 & 250 & 5 & 11 \\
\hline Lina $A_{3}$ & 100 & 50 & 5 & 2 \\
\hline Kakim $A_{4}$ & 208 & 100 & 5 & 4 \\
\hline
\end{tabular}

Capacity elevators (silos)

\begin{tabular}{|c|c|}
\hline Elevators & Capacity $(\mathrm{t})$ \\
\hline I $B_{1}$ & 250 \\
\hline II $B_{2}$ & 250 \\
\hline III $B_{3}$ & 300 \\
\hline
\end{tabular}


Analytical and graphical methods for...

Distance from the point of departure to a point on the matter

T a ble 3

\begin{tabular}{|c|c|c|c|c|}
\hline $\begin{array}{c}\text { Distance }(\mathrm{km}) \\
\text { there and back) }\end{array}$ & $\begin{array}{c}\text { Fuel } \\
\text { consumption }(\mathrm{L})\end{array}$ & $\begin{array}{c}\text { The point of } \\
\text { departure } A_{i} \text { and } \\
\text { destination } B_{j}\end{array}$ & $\begin{array}{c}\text { The cost of } \\
\text { fuel for } 1 \text { flight } \\
\left(y_{x} 115\right) \text { tg }\end{array}$ & $\begin{array}{c}\text { Total transportation } \\
\text { costs for } 1 \\
\text { flight } 1)+2)+3)\end{array}$ \\
\hline 56 & 22,4 & $A_{3} B_{1}$ & 2587 & $7191 \approx 72$ \\
\hline 60 & 24 & $A_{1} B_{1}$ and $A_{4} B_{3}$ & 2760 & $7374 \approx 74$ \\
\hline 62 & 24,8 & $A_{4} B_{1}$ and $A_{3} B_{3}$ & 2842 & $7456 \approx 75$ \\
\hline 66 & 26,4 & $A_{1} B_{2}$ and $A_{3} B_{2}$ & 3036 & $7650 \approx 77$ \\
\hline 68 & 27,2 & $A_{2} B_{2}$ & 3128 & $7742 \approx 77$ \\
\hline 70 & 28 & $A_{1} B_{3}$ and $A_{2} B_{3}$ & 3225 & $7839 \approx 78$ \\
\hline 72 & 28,8 & $A_{2} B_{1}$ & 3312 & $7926 \approx 79$ \\
\hline 74 & 29,4 & $A_{4} B_{2}$ & 3404 & $8018 \approx 80$ \\
\hline
\end{tabular}

$$
\begin{gathered}
(A)=\left\{\begin{array}{l}
\left|A_{1} B_{1}\right|=30 \mathrm{~km}, \\
\left|A_{1} B_{2}\right|=33 \mathrm{~km}, \\
\left|A_{1} B_{3}\right|=35 \mathrm{~km} .
\end{array} \quad(S)=\left\{\begin{array}{l}
\left|A_{2} B_{1}\right|=36 \mathrm{~km}, \\
\left|A_{2} B_{2}\right|=34 \mathrm{~km}, \\
\left|A_{2} B_{3}\right|=35 \mathrm{~km} .
\end{array}\right.\right. \\
(L)=\left\{\begin{array}{l}
\left|A_{3} B_{1}\right|=28 \mathrm{~km}, \\
\left|A_{3} B_{2}\right|=33 \mathrm{~km}, \\
\left|A_{3} B_{3}\right|=32 \mathrm{~km} .
\end{array} \quad(K)=\left\{\begin{array}{l}
\left|A_{4} B_{1}\right|=28 \mathrm{~km}, \\
\left|A_{4} B_{2}\right|=37 \mathrm{~km}, \\
\left|A_{4} B_{3}\right|=30 \mathrm{~km} .
\end{array}\right.\right.
\end{gathered}
$$

Transportation cost:

$$
C=\left(\begin{array}{lll}
74 & 77 & 78 \\
79 & 77 & 78 \\
72 & 77 & 75 \\
75 & 80 & 74
\end{array}\right)
$$

Volume of cargo at points of departure.

For transportation of used Kamaz trailer with a load capacity of $12+12=24 \mathrm{~m}$.

This kind of transport for $1 \mathrm{~km}$ consumes 0.4 liters of fuel.

The cost (price) of fuel (kerosene) per 1 liter 115 tenge.

Depreciation and driver's salary for one flight (round trip) is 4614 tenge.

There is fuel consumption table depending on the distance from the $A_{i}$ to $B_{j}$ to and from $B_{j}$ to $A_{i}$ (back and forth) and determination the cost of transportation for one flight (Table 4).

\begin{tabular}{|c|c|c|c|c|}
\hline \multirow{2}{*}{ Suppliers } & \multicolumn{3}{|c|}{ Consumers (granary) } & \multirow{2}{*}{ Stock } \\
\hline & $I_{\left(\beta_{1}\right)} \beta_{1}$ & & $I I I_{\left(\beta_{8}\right)} \beta_{3}$ & \\
\hline $\begin{array}{ll}\text { Aiman } & A_{1} \\
& \alpha_{1}\end{array}$ & $\begin{array}{r}10,5 \mathrm{p} . \\
250 \\
\end{array}$ & $\begin{array}{lr} & 6,5 \mathrm{p} \\
& \\
& 150\end{array}$ & 78 & 400 \\
\hline $\begin{array}{rr}\text { Sharipa } & A_{2} \\
& \alpha_{2}\end{array}$ & 79 & $\begin{array}{ll} & 4 \text { p. } \\
77 & \\
& 100 \\
\end{array}$ & $\begin{array}{cc} & 6,5 \mathrm{p} . \\
-\quad 180\end{array}$ & 250 \\
\hline $\begin{array}{ll}\text { Lina } & A_{3} \\
& \alpha_{3}\end{array}$ & 72 & 77 & $\begin{array}{ll}78 & 2 \mathrm{p} \\
78 & \\
& 50\end{array}$ & 50 \\
\hline $\begin{array}{cc}\text { Kakim } & A_{4} \\
& \alpha_{4}\end{array}$ & 75 & 80 & $74_{100}^{4 p}$ & 100 \\
\hline Needs & 250 & 250 & 300 & $\longleftarrow 800$ \\
\hline
\end{tabular}

The first reference transportation plan by building a north-west corner 
Total cost of transportation:

$$
\sum_{i=1, j=1}^{4.3} c_{i j} .
$$

$x_{i j}=10,5 \cdot 74+6,5 \cdot 77+4 \cdot 77+6,5 \cdot 78+2 \cdot 78+4 \cdot 74=777+500,5+308+507+156+296=2544,5(254450 t g)$.

We verify the plan for optimality. To determine the true value of potential we have to decide the following uncertain system.

$$
\begin{gathered}
\left\{\begin{array}{l}
\alpha_{1}+\beta_{1}=74 ; \\
\alpha_{1}+\beta_{2}=77 ; \\
\alpha_{2}+\beta_{2}=77 ; \\
\alpha_{2}+\beta_{3}=78 ; \\
\alpha_{3}+\beta_{3}=78 ; \\
\alpha_{4}+\beta_{3}=74
\end{array}\right. \\
\alpha_{1}=0 ; \quad \beta_{1}=74 ; \\
\alpha_{2}=0 ; \quad \beta_{2}=77 ; \\
\alpha_{3}=0 ; \quad \beta_{3}=78 ; \\
\alpha_{4}=-4 .
\end{gathered}
$$

Defining the potential indirect value.

$$
\begin{gathered}
c_{13}^{\prime}=\alpha_{1}+\beta_{3}=78=78=c_{13}+ \\
c_{21}^{\prime}=\alpha_{2}+\beta_{1}=74<79=c_{21}+ \\
c_{31}^{\prime}=\alpha_{3}+\beta_{1}=74>72=c_{31}- \\
c_{32}^{\prime}=\alpha_{3}+\beta_{2}=77=77=c_{32}+ \\
c_{41}^{\prime}=\alpha_{4}+\beta_{1}=-4+77=70<75=c_{41}+ \\
c_{42}^{\prime}=\alpha_{4}+\beta_{3}=-4+77=73<80=c_{42}+
\end{gathered}
$$

This plan is not optimal, because the cell $\left(A_{3} B_{1}\right)$ indirect value potential $c_{31}^{\prime}$ greater than the true value $c_{31} \cdot\left(c_{31}^{\prime}>c_{31}\right)$

Building optimal plan transport method of potentials [1].

Building the cycle.

$x_{31} x_{33} x_{23} x_{22} x_{12} x_{11} x_{31}$

$+-+-+-+$

Defining element:

$x=\min \left\{x_{33}, x_{22}, x_{11}\right\}=\min \{50,100,250\}=50$.

Then,

$x_{31}^{\prime}=x_{31}+x=0+50=50$.

$x_{33}^{\prime}=x_{33}-x=50-50=0$.

$x_{23}^{\prime}=x_{23}+x=150+50=200$.

$x_{22}^{\prime}=x_{22}-x=100-50=50$.

$x_{12}^{\prime}=x_{12}+x=150+50=200$.

$x_{11}^{\prime}=x_{11}-x=250-50=200$.

The new transportation plan Table 5 is as follows: 


\section{Transportation plan}

\begin{tabular}{|c|c|c|c|c|}
\hline \multirow[t]{2}{*}{ Suppliers } & \multicolumn{3}{|c|}{ Consumers (granary) } & \multirow[t]{2}{*}{ Stock } \\
\hline & $\begin{array}{ll}I & \beta_{1}\end{array}$ & $\begin{array}{ll}I I & \beta_{2}\end{array}$ & III $\beta_{3}$ & \\
\hline $\begin{aligned} \text { Aiman } & A_{1} \\
& \alpha_{1}\end{aligned}$ & $\begin{array}{ll} & 8 \mathrm{p} . \\
74 & \\
& 200\end{array}$ & $\begin{array}{ll}77 & 8,5 \mathrm{p} . \\
& 200\end{array}$ & 78 & 400 \\
\hline $\begin{array}{r}\text { Sharipa } A_{2} \\
\alpha_{2}\end{array}$ & 79 & $\begin{array}{ll} & 2 \text { p. } \\
77 & \\
& 50 \\
\end{array}$ & $\begin{array}{ll}78 & 2,5 \mathrm{p} . \\
& 200 \\
\end{array}$ & 250 \\
\hline $\begin{array}{cc}\text { Lina } & A_{3} \\
& \alpha_{3}\end{array}$ & $72 \quad 2$ p. & 77 & 78 & 50 \\
\hline $\begin{array}{rr}\text { Kakim } & A_{4} \\
& \alpha_{4}\end{array}$ & 75 & 80 & $\begin{array}{ll} & 4 \mathrm{p} . \\
74 & \\
& 100 \\
\end{array}$ & 100 \\
\hline Needs & 250 & 250 & 300 & $\longleftarrow 800$ \\
\hline
\end{tabular}

Verify the plan for optimality.

$$
\begin{gathered}
\left\{\begin{array}{l}
\alpha_{1}+\beta_{1}=74 ; \\
\alpha_{1}+\beta_{2}=77 ; \\
\alpha_{2}+\beta_{2}=77 ; \\
\alpha_{2}+\beta_{3}=78 ; \\
\alpha_{3}+\beta_{3}=78 ; \\
\alpha_{4}+\beta_{3}=74 .
\end{array}\right. \\
\alpha_{1}=0 ; \quad \beta_{1}=74 ; \\
\alpha_{2}=0 ; \quad \beta_{2}=77 ; \\
\alpha_{3}=0 ; \quad \beta_{3}=78 ; \\
\alpha_{4}=-4 .
\end{gathered}
$$

We verify the plan for optimality and define the potential indirect value.

$$
\begin{gathered}
c_{13}^{\prime}=\alpha_{1}+\beta_{3}=78=78=c_{13}+ \\
c_{21}^{\prime}=\alpha_{2}+\beta_{1}=74<79=c_{21}+ \\
c_{31}^{\prime}=\alpha_{3}+\beta_{1}=74>72=c_{31}- \\
c_{32}^{\prime}=\alpha_{3}+\beta_{2}=77=77=c_{32}+ \\
c_{41}^{\prime}=\alpha_{4}+\beta_{1}=-4+77=70<75=c_{41}+ \\
c_{42}^{\prime}=\alpha_{4}+\beta_{3}=-4+77=73<80=c_{42}+
\end{gathered}
$$

In all unoccupied cells indirect value potential greater than the true value, $c_{i j}^{\prime} \leq c_{i j}$.

Total cost of transportation.

$S=8 \cdot 74+8,5 \cdot 77+2 \cdot 77+2,5 \cdot 78+2 \cdot 72+4 \cdot 74=592+654,5+154+195+144+296=2035,5(203550$ tg).

Potential method improved the original plan, and the total cost of transportation decreased by $254450-203550=50900$ tg. Comment. 
The volume of cargo at points of departure are $A_{i}$ and capacity of elevators, we used to build Tables ( 1 and 2) distribution transportation. To determine the total cost of transportation have used the number of flights from $A_{i}$ in $B_{j}$ and transportation cost.

$$
S=\sum_{i=1, j=1}^{3,4} c_{i j} \cdot k_{i j},
$$

where $k_{i j}$ - the number of flights from $A_{i}$ в $B_{j}$ (roundtrip); $c_{i j}$ - cost per flight (roundtrip) Graphic illustration of this problem can be given with the help of graphs. Then the method of «bypassing Count» can be used to solve this problem. (Tarry algorithm). (Fig. 1-3).

(A) $A_{1}$

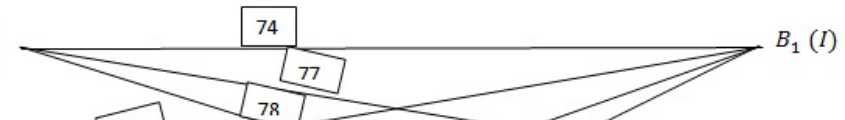

(Sh) $A_{2}$

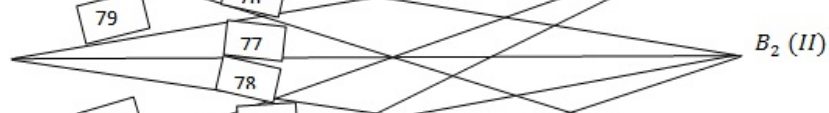

(L) $A_{3}$
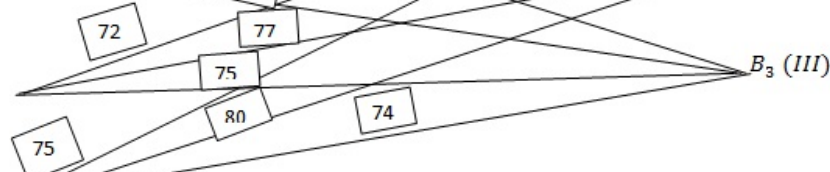

(K) $A_{4}$

Figure 1. Count the total distribution of transportation, where $A_{1}, A_{2}, A_{3}, A_{4}$ - the points of departure (grain farms), $B_{1}, B_{2}, B_{3}$ - Destinations (granary)

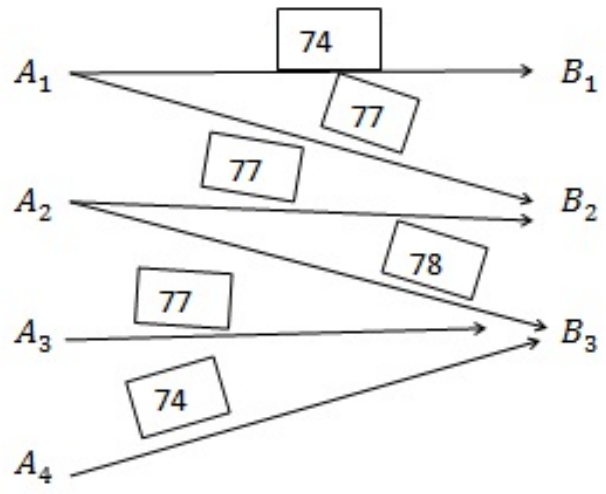

Figure 2. The graph corresponding to the first reference plane

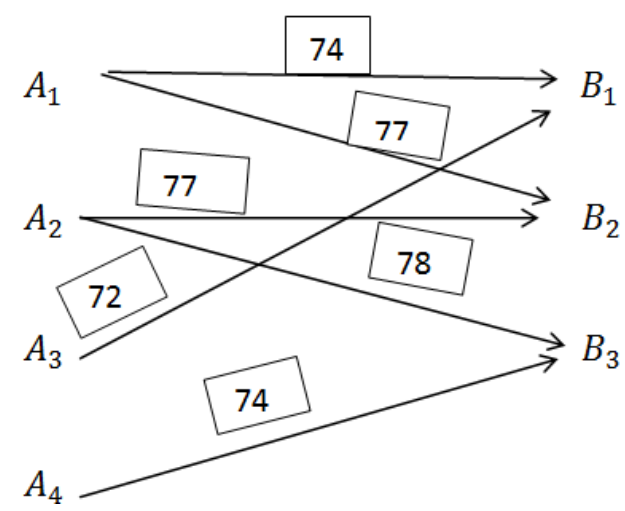

Figure 3. The graph corresponding to the second (optimal) distribution plan 
These graphs are determined by the composition of graphs $K_{4}$ and $K_{3}$.

$$
K_{4} \cdot K_{3}=G .
$$

As a result, we obtain a bipartite graph with weights.

The vertices $K_{4}-A_{1}, A_{2}, A_{3}, A_{4}$ - manufacturers.

The vertices - manufacturers.

The vertices $K_{3}-B_{1}, B_{2}, B_{3}$ - consumers.

The vertices - consumers.

Count $G=K_{4} \cdot K_{3}$ is also a weighted graph, where the weights are the elements of $S$.

Each arc $A_{i} B_{j}$ directed graph $G$ corresponds to the weight $c_{i} j \in C_{0}$

\title{
References
}

1 Song Qiang, Gao Xuexia, Santos Emmanuel T. International Journal Of Bifurcation And Chaos. - 2015. - Vol. 25, No. 14. - P. 1540031.

2 Щербаков В.В. Основы логистики (теория и практика) / В.В. Щербаков, И.Л.Киппер, Л.А.Мясникова. - СПб.: Питер Пресс, 2009. - 426 с.

3 Просветов Г.И. Математические методы в логистике: задачи и решения: учеб.-практ. пособие / Г.И.Просветов. - М.: Альфа-Пресс, 2008. - 302 с.

4 Акулич И.Л. Математическое программирование в примерах и задачах / И.Л.Акулич. - М.: Высш. шк., 1986. - 317 с.

\author{
Қ. Жетпісов, Н.Г. Карбенова
}

\section{Транспорттық логистиканың бір проблемасын шешудің аналитикалық және графиктік әдістері}

\begin{abstract}
Мақалада нақты транспорттық логистика есебін потенциалдар әдісімен шешудің жолы көрсетілген. Алғашқы тірек жоспары солтүстік-батыс бұрышы әдісімен құрылған. Сонымен қатар есептік графиктердің көмегімен шешу әдісі де берілген. Авторлармен Қостанай облысы (Арқалық қ.) шаруа қожалығы мысалында көліктік логистика есебінің оңтайлы шешімі көрсетілген.

Kiлm сөздер: логистика, көліктік тапсырма, потенциал, солтүстік-батыс бұрышы, санау, екі жақты санау, құндылық, көлем.
\end{abstract}

\author{
К. Жетписов, Н.Г. Карбенова
}

\section{Аналитический и графический методы решения одной проблемы транспортной логистики}

В статье показан способ решения конкретной задачи транспортной логистики методом потенциалов. Первый опорный план построен методом северо-западного угла. Кроме того, показан графический способ решения задачи. Авторами дан оптимальный метод решения задачи транспортной логистики на примере реального хозяйства Костанайской области (г. Аркалык).

Ключевые слова: логистика, транспортная задача, потенциальный, северо-западный угол, подсчет, двухсторонний счет, стоимость, объем. 


\section{References}

1 Song Qiang, Gao, Xuexia \& Santos, Emmanuel T. (2015). International Journal Of Bifurcation And Chaos. Vol. 25, 14.

2 Sherbakov, V.V., Kipper, I.L. \& Miasnikova, L.A. (2009). Osnovy lohistiki (teoriia i praktika) [Fundamentals of Logistics (theory and practice)]. Saint Petersburg: Piter Press [in Russian].

3 Prosovetov, G.I. (2008). Matematicheskie metody v lohistike: zadachi i resheniia: uchebno-prakticheskoe posobie [Mathematical Methods in Logistics: Challenges and Solutions teaching practical manual]. Moscow: Alfa-Press [in Russian].

4 Akulich, I.L. (1986). Matematicheskoe prohrammirovanie v primerakh i zadachakh /Mathematical programming examples and problems]. Moscow: Vysshaia shkola [in Russian]. 